\title{
A Short Proof of the Hölder-Poincaré Duality for $L_{p}$-Cohomology
}

\author{
Vladimir Gol'dshtein (*) - Marc Troyanov (**)
}

ABSTRACT - We give a short proof of the duality theorem for the reduced $L_{p}$-cohomology of a complete oriented Riemannian manifold.

Let $(M, g)$ be an oriented Riemannian manifold. For any $1 \leq p<\infty$ we denote by $L^{p}\left(M, \Lambda^{k}\right)$ the space of $p$-integrable differential forms on $M$. An element of that space is a measurable differential $k$-form $\omega$ such that

$$
\|\omega\|_{p}:=\left(\int_{M}|\omega|_{x}^{p} d \operatorname{vol}_{g}(x)\right)^{1 / p}<\infty .
$$

Recall that a differential form $\theta \in L^{p}\left(M, \Lambda^{k+1}\right)$ is the weak exterior differential of the form $\phi \in L^{p}\left(M, \Lambda^{k}\right)$ if one has

$$
\int_{M} \theta \wedge \omega=(-1)^{k+1} \int_{M} \phi \wedge d \omega
$$

for any $\omega \in \mathcal{D}^{n-k}(M)$, where $\mathcal{D}^{m}(M)$ denotes the vector space of smooth differential $m$-forms with compact support in $M$.

One writes $d \phi=\theta$ if $\theta$ is the weak exterior differential of $\phi$ and $Z_{p}^{k}(M)=\operatorname{ker} d \cap L^{p}\left(M, \Lambda^{k}\right)$ denotes the set of weakly closed forms in $L^{p}\left(M, \Lambda^{k}\right)$. It is easy to check that $Z_{p}^{k}(M)$ is a closed linear subspace of

(*) Indirizzo dell'A.: Department of Mathematics, Ben Gurion University of the Negev, P.O.Box 653, Beer Sheva, Israel.

E-mail: vladimir@bgumail.bgu.ac.il

(**) Indirizzo dell'A.: Section de Mathematiques, École Polytechnique Féderale de Lausanne, 1015 Lausanne - Switzerland.

E-mail: marc.troyanov@epfl.ch

AMS Mathematics Subject Classification: 58A10, 58A12, 53c. 
$L^{p}\left(M, \Lambda^{k}\right)$, in particular it is a Banach space (see [5, Lemma 2.2]). We then introduce the space

$$
B_{p}^{k}(M)=d\left(L^{p}\left(M, \Lambda^{k-1}\right)\right) \cap L^{p}\left(M, \Lambda^{k}\right)
$$

of exact $L^{p}$-forms and we shall denote by $\bar{B}_{p}^{k}(M)$ the closure of $B_{p}^{k}(M)$ in $L^{p}\left(M, \Lambda^{k}\right)$. Because $Z_{p}^{k}(M) \subseteq L^{p}\left(M, \Lambda^{k}\right)$ is a closed subspace and $d \circ d=0$, we have $\bar{B}_{p}^{k}(M) \subseteq Z_{p}^{k}(M)$. The reduced $L_{p}$-cohomology of $(M, g)$ (where $1 \leq p<\infty)$ is defined to be the quotient

$$
\bar{H}_{p}^{k}(M)=Z_{p}^{k}(M) / \bar{B}_{p}^{k}(M)
$$

This is a Banach space for the natural (quotient) norm and the goal of this paper is to prove the following Theorem (here and throughout the paper, $p^{\prime}=p /(p-1)$ is the conjugate number of $\left.p\right)$.

Duality Theorem. Let $(M, g)$ be a complete oriented Riemannian manifold of dimension $n$ and $1<p<\infty$. Then $\bar{H}_{p}^{k}(M)$ is isometric to the dual of $\bar{H}_{p^{\prime}}^{n-k}(M)$. The duality is given by the integration pairing:

$$
\begin{aligned}
\bar{H}_{p}^{k}(M) \times \bar{H}_{p^{\prime}}^{n-k}(M) & \rightarrow \quad \mathbb{R} \\
([\omega],[\theta]) & \mapsto \int_{M} \omega \wedge \theta .
\end{aligned}
$$

REMARK. By "the dual space" $X^{\prime}$ of a Banach space $X$, we of course mean the topological dual, i.e. the vector space of continuous linear functionals together with its natural norm. The isomorphism between $\bar{H}_{p}^{k}(M)$ and the dual of $\bar{H}_{p^{\prime}}^{n-k}(M)$ has first been proved in 1986 by V. M. Gol'dshtein, V.I. Kuz'minov and I.A. Shvedov, see [4]. In fact that paper also describes the dual space to the $L_{p}$-cohomology of non complete manifolds. The proof we present here is simpler and more direct than the proof in [4], although it doesn't seem to be extendable to the non complete case. Note that this duality theorem is useful to prove vanishing or non vanishing results in $L_{p^{-}}$ cohomology, see e.g. [5, 7, 8].

Let us also mention that Gromov deduced the above theorem from the simplicial version of the $L_{p}$-cohomology, see [7]. Gromov's argument works only for Riemannian manifolds with bounded geometry, while the proof we give here works for any complete manifold. Our proof can also be extended to the more general $L_{q, p}$-cohomology, see [6].

The proof will rest on a few auxiliary facts. Recall first that a pairing between two Banach spaces $X_{0}$ and $X_{1}$ is simply a continuous bilinear map 
$I: X_{0} \times X_{1} \rightarrow \mathbb{R}$. Such a pairing defines two continuous linear maps $\lambda: X_{0} \rightarrow X_{1}^{\prime}$, and $\mu: X_{1} \rightarrow X_{0}^{\prime}$ defined by

$$
\lambda_{\xi}(\eta)=\mu_{\eta}(\xi)=I(\xi, \eta)
$$

for any $\xi \in X_{0}$ and $\eta \in X_{1}$.

Definition 1. An isometric duality between two Banach spaces $X_{0}$ and $X_{1}$ is a pairing $I: X_{0} \times X_{1} \rightarrow \mathbb{R}$ such that the associated maps $\lambda: X_{0} \rightarrow X_{1}^{\prime}$, and $\mu: X_{1} \rightarrow X_{0}^{\prime}$ are bijective isometries.

Observe that if an isometric duality exists between two Banach spaces, then these spaces are reflexive. The classic $L^{p}-L^{p^{\prime}}$ duality for function spaces extends to the case of differential forms, see [4]:

Proposition 2. If $1<p<\infty$, then the pairing $L^{p}\left(M, \Lambda^{k}\right) \times$ $\times L^{p^{\prime}}\left(M, \Lambda^{n-k}\right) \rightarrow \mathbb{R}$ defined by

$$
\langle\omega, \varphi\rangle=\int_{M} \omega \wedge \varphi
$$

is an isometric duality. In particular, $L^{p}\left(M, \Lambda^{k}\right)$ is a reflexive Banach space.

We will also need the following density result whose proof is based on regularization methods, see e.g. [3, 5]:

Proposition 3. Let $\theta \in L^{p}\left(M, \Lambda^{k-1}\right)$ be a $(k-1)$-form whose weak exterior differential is p-integrable, $d \theta \in L^{p}\left(M, \Lambda^{k}\right)$. Then there exists a sequence $\theta_{j} \in C^{\infty}\left(M, \Lambda^{k-1}\right)$ such that $\theta=\lim _{j \rightarrow \infty} \theta_{j}$ and $d \theta=\lim _{j \rightarrow \infty} d \theta_{j}$ in $L^{p}(M)$.

The next lemma is the place where the completeness hypothesis enters:

LEMma 4. If $(M, g)$ is complete, then $d \mathcal{D}^{k-1}(M)$ is dense in $B_{p}^{k}(M)$.

Proof. Because $M$ is complete, one can find a sequence of smooth functions with compact support $\left\{\eta_{j}\right\} \subseteq C_{0}^{\infty}(M)$ such that $0 \leq \eta_{j} \leq 1$, $\lim \sup \left|d \eta_{j}\right|=0$ and $\eta_{j} \rightarrow 1$ uniformly on every compact subset of $M$. Let

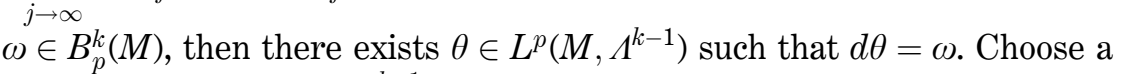
sequence $\left\{\theta_{j}\right\} \subseteq C^{\infty}\left(M, \Lambda^{k-1}\right)$ as in Proposition 3, i.e. $\theta_{j} \rightarrow \theta$ and $d \theta_{j} \rightarrow d \theta=$ 
$=\omega$ in $L^{p}(M)$ and set $\tilde{\theta}_{j}=\eta_{j} \theta_{j} \in \mathcal{D}^{k-1}(M)$. We first claim that $\left(\tilde{\theta}_{j}-\theta_{j}\right) \rightarrow 0$ in $L^{p}\left(M, \Lambda^{k-1}\right)$. Indeed, fix $\varepsilon>0$ and choose a compact set $Q$ such that $\|\theta\|_{L^{p}(M \backslash Q)}<\varepsilon$. Since $\left|\eta_{j}-1\right|<1$, we have

$$
\begin{aligned}
\left\|\tilde{\theta}_{j}-\theta_{j}\right\|_{L^{p}(M)} & \leq\left\|\left(\eta_{j}-1\right) \theta_{j}\right\|_{L^{p}(Q)}+\left\|\theta_{j}\right\|_{L^{p}(M \backslash Q)} \\
& \leq\left\|\left(\eta_{j}-1\right) \theta_{j}\right\|_{L^{p}(Q)}+\left\|\theta_{j}-\theta\right\|_{L^{p}(M \backslash Q)}+\|\theta\|_{L^{p}(M \backslash Q)} .
\end{aligned}
$$

The first term converges to zero because $\eta_{j} \rightarrow 1$ uniformly on $Q$ and $\left\{\left\|\theta_{j}\right\|_{L^{p}}\right\}$ is bounded. The second term converges to zero because $\theta_{j} \rightarrow \theta$ in $L^{p}\left(M, \Lambda^{k-1}\right)$ and the last term is bounded by $\varepsilon$, hence

$$
\limsup _{j \rightarrow \infty}\left\|\tilde{\theta}_{j}-\theta_{j}\right\|_{L^{p}(M)} \leq \varepsilon .
$$

Since $\varepsilon$ is arbitrary, the limit is zero and we obtain

$$
\lim _{j \rightarrow \infty}\left\|\tilde{\theta}_{j}-\theta\right\|_{L^{p}(M)} \leq \lim _{j \rightarrow \infty}\left\|\tilde{\theta}_{j}-\theta_{j}\right\|_{L^{p}(M)}+\lim _{j \rightarrow \infty}\left\|\theta_{j}-\theta\right\|_{L^{p}(M)}=0 .
$$

We similarly have

$$
\begin{aligned}
\left\|d \tilde{\theta}_{j}-d \theta_{j}\right\|_{p} & \leq\left\|\left(\eta_{j}-1\right) d \theta_{j}\right\|_{p}+\left\|d \eta_{j} \wedge \theta_{j}\right\|_{p} \\
& \leq\left\|\left(\eta_{j}-1\right) d \theta_{j}\right\|_{p}+\sup \left|d \eta_{j}\right| \cdot\left\|\theta_{j}\right\|_{p} \rightarrow 0 .
\end{aligned}
$$

This implies that $\omega=\lim _{j \rightarrow \infty} d \tilde{\theta}_{j}$ in $L^{p}$.

Definition 5. Given an isometric duality $I: X_{0} \times X_{1} \rightarrow \mathbb{R}$ and a nonempty subset $B$ of $X_{0}$, we define the annihilator $B^{\perp} \subseteq X_{1}$ of $B$ to be the set of all elements $\eta \in X_{1}$ such that $I(\xi, \eta)=0$ for all $\xi \in B$.

For any $B \subseteq X_{0}$ the annihilator $B^{\perp}$ is a closed linear subspace of $X_{1}$. The Hahn-Banach Theorem implies that if $B$ is a linear subspace of $X_{0}$ then $\left(B^{\perp}\right)^{\perp}=\bar{B}$.

For these and further facts on the notion of annihilator, we refer to the books $[1,2]$.

The proof of the duality Theorem is based on the following lemma about annihilators:

Lemma 6. Let $I: X_{0} \times X_{1} \rightarrow \mathbb{R}$ be an isometric duality between two Banach spaces. Let $B_{0}, A_{0}, B_{1}, A_{1}$ be linear subspaces such that

$$
B_{0} \subseteq A_{0}=B_{1}^{\perp} \subseteq X_{0} \quad \text { and } \quad B_{1} \subseteq A_{1}=B_{0}^{\perp} \subseteq X_{1} .
$$

Then the pairing $\bar{I}: \bar{H}_{0} \times \bar{H}_{1} \rightarrow \mathbb{R}$ of $\bar{H}_{0}:=A_{0} / \bar{B}_{0}$ and $\bar{H}_{1}:=A_{1} / \bar{B}_{1}$ is well defined and induces an isometric duality between $\bar{H}_{0}$ and $\bar{H}_{1}$. 
Proof. Observe first that $A_{i} \subseteq X_{i}$ is a closed subspace since the annihilator of any subset of a Banach space is always a closed linear subspace.

The bounded bilinear map $I: A_{0} \times A_{1} \rightarrow \mathbb{R}$ is defined by restriction. It gives rise to a well defined bounded bilinear map $\bar{I}: A_{0} / \bar{B}_{0} \times A_{1} / \bar{B}_{1} \rightarrow \mathbb{R}$ because we have the inclusions $B_{0} \subseteq B_{1}^{\perp}$ and $B_{1} \subseteq B_{0}^{\perp}$.

We denote by $\lambda: X_{0} \rightarrow X_{1}^{\prime}$ the isometry induced by the pairing $I$, by $\bar{\lambda}: \bar{H}_{0} \rightarrow \bar{H}_{1}^{\prime}$ the map defined by the pairing $\bar{I}$ and by $\pi_{i}: A_{i} \rightarrow \bar{H}_{i}$ $(i=1,2)$, the canonical projections.

We first prove that $\left\|\bar{\lambda}_{\xi}\right\|_{\bar{H}_{1}^{\prime}} \leq\|\xi\|_{\bar{H}_{0}}$ for any $\xi \in \bar{H}_{0}$. Indeed, let us choose $\hat{\xi} \in A_{0}$ such that $\pi_{0}(\hat{\xi})=\xi$, we have

$$
\begin{aligned}
\left\|\bar{\lambda}_{\xi}\right\|_{\bar{H}_{1}^{\prime}} & =\sup \left\{\bar{I}(\xi, \eta) \mid \eta \in \bar{H}_{1},\|\eta\|_{\bar{H}_{1}} \leq 1\right\} \\
& \leq \sup \left\{I(\hat{\xi}, \hat{\eta}) \mid \hat{\eta} \in A_{1},\|\hat{\eta}\|_{A_{1}} \leq 1\right\} \\
& \leq \sup \left\{I(\hat{\xi}, \hat{\eta}) \mid \hat{\eta} \in X_{1},\|\hat{\eta}\|_{X_{1}} \leq 1\right\}=\left\|\lambda_{\hat{\xi}}\right\|_{X_{1}^{\prime}} .
\end{aligned}
$$

By hypothesis, we have $\left\|\lambda_{\hat{\xi}}\right\|_{X_{1}^{\prime}}=\|\hat{\xi}\|_{X_{0}}$, therefore

$$
\|\xi\|_{\bar{H}_{0}}=\inf _{\hat{\xi} \in \pi_{0}^{-1}(\xi)}\|\hat{\xi}\|_{X_{0}}=\inf _{\hat{\xi} \in \pi_{0}^{-1}(\xi)}\left\|\lambda_{\xi}\right\|_{X_{1}^{\prime}} \geq\left\|\bar{\lambda}_{\xi}\right\|_{\bar{H}_{1}^{\prime}}
$$

We then prove that for any $\theta \in \bar{H}_{1}^{\prime}$, there exists an element $\xi \in \bar{H}_{0}$ such that $\theta=\bar{\lambda}_{\xi}$ and $\|\theta\|_{\bar{H}_{1}^{\prime}} \geq\|\xi\|_{\bar{H}_{0}}$. This implies that $\bar{\lambda}$ is surjective and $\left\|\bar{\lambda}_{\xi}\right\|_{\bar{H}_{1}^{\prime}} \geq\|\xi\|_{\bar{H}_{0}}$.

Indeed, for any $\theta \in \bar{H}_{1}^{\prime}$, the linear form $\hat{\theta}=\theta \circ \pi_{1}: A_{1} \rightarrow \mathbb{R}$ satisfies $\hat{\theta}(b)=0$ for any $b \in B_{1}$ and $\|\hat{\theta}\|_{A_{1}^{\prime}}=\|\theta\|_{\bar{H}_{1}^{\prime}}$. By the Hahn-Banach Theorem, there exists a continuous extension $\hat{\varphi}: X_{1} \rightarrow \mathbb{R}$ of $\hat{\theta}$ such that $\|\hat{\varphi}\|_{X_{1}^{\prime}}=\|\hat{\theta}\|_{A_{1}^{\prime}}$. Since $\lambda: X_{0} \rightarrow X_{1}^{\prime}$ is an isometry, one can find $\hat{\xi} \in X_{0}$ such that $\lambda_{\hat{\xi}}=\hat{\varphi}$ and

$$
\|\hat{\xi}\|_{X_{0}}=\|\hat{\varphi}\|_{X_{1}^{\prime}}=\|\hat{\theta}\|_{A_{1}^{\prime}}=\|\theta\|_{\bar{H}_{1}^{\prime}}
$$

For any $b \in B_{1}$, we have $I(\hat{\xi}, b)=\lambda_{\hat{\xi}}(b)=\hat{\theta}(b)=0$, thus $\hat{\xi} \in B_{1}^{\perp}=A_{0}$. Let us set $\xi=\pi_{0}(\hat{\xi})$, we have

$$
\bar{I}(\xi, \eta)=I(\hat{\xi}, \hat{\eta})=\hat{\theta}(\hat{\eta})=\theta(\eta)
$$

for any $\eta \in \bar{H}_{1}$ and $\hat{\eta} \in A_{1}$, that is $\theta=\bar{\lambda}_{\xi}$. We also have

$$
\|\xi\|_{\bar{H}_{0}} \leq\|\hat{\xi}\|_{X_{0}}=\|\theta\|_{\bar{H}_{1}^{\prime}}=\left\|\bar{\lambda}_{\xi}\right\|_{\bar{H}_{1}^{\prime}} .
$$

In conclusion, we have have proved that $\bar{\lambda}: \bar{H}_{0} \rightarrow \bar{H}_{1}^{\prime}$ is norm preserving and surjective: it is an isometry. The proof that $\bar{\mu}: \bar{H}_{1} \rightarrow \bar{H}_{0}^{\prime}$ is also an isometry is the same. 
Proof of the Duality Theorem. Let $\phi \in L^{p}\left(M, \Lambda^{k}\right)$, then $d \phi=0$ in the weak sense if and only if $\int_{M} \phi \wedge d \omega=0$ for any $\omega \in \mathcal{D}^{n-k-1}(M)$. This precisely means that $Z_{p}^{k}(M) \subseteq L^{p}\left(M, \Lambda^{k}\right)$ is the annihilator of $d \mathcal{D}^{n-k-1}(M) \subseteq$ $\subseteq L^{p^{\prime}}\left(M, \Lambda^{n-k}\right)$ for the pairing (1):

$$
Z_{p}^{k}(M)=\left(d \mathcal{D}^{n-k-1}\right)^{\perp}(M) .
$$

By lemma $1, d \mathcal{D}^{n-k-1}(M)$ and $B_{p^{\prime}}^{n-k}$ have the same annihilator, thus

$$
B_{p}^{k} \subseteq Z_{p}^{k}=\left(B_{p^{\prime}}^{n-k}\right)^{\perp} \subseteq L^{p}\left(M, \Lambda^{k}\right) .
$$

Similarly, we also have

$$
B_{p^{\prime}}^{n-k} \subseteq Z_{p^{\prime}}^{n-k}=\left(B_{p}^{k}\right)^{\perp} \subseteq L^{p^{\prime}}\left(M, \Lambda^{n-k}\right),
$$

and Lemma 1 says that the duality (1) induces an isometric duality between $Z_{p^{\prime}}^{n-k} / \bar{B}_{p^{\prime}}^{n-k}$ and $Z_{p}^{k} / \bar{B}_{p}^{k}$.

Acknowledgments. We thank the anonymous referee for helpful comments and for pointing out that the duality in our main theorem is an isometric duality.

\section{REFERENCES}

[1] H. BRezIs, Analyse fonctionnelle, Théorie et applications. Dunod, Paris 1999.

[2] J. ConwaY, A course in functional analysis. Second edition. Graduate Texts in Mathematics, 96. Springer-Verlag, New York, 1990.

[3] V. M. Gol'dshtein - V. I. Kuz'Minov - I. A. Shvedov, A Property of de Rham Regularization Operators, Siberian Math. Journal, 25, No 2 (1984).

[4] V. M. Gol'DShTEIN - V. I. KuZ'Minov - I. A. ShVEdov, Dual spaces of Spaces of Differential Forms, Siberian Math. Journal, 54, No 1 (1986).

[5] V. Gol'DSHTEIn - M. TroYANov, Sobolev Inequality for Differential forms and $L_{p}$-cohomology. Journal of Geom. Anal., 16, No 4 (2006), pp. 597-631.

[6] V. Gol'Dshtein - M. Troyanov, The Hölder-Poincaré Duality for $L_{q, p^{-}}$ cohomology Preprint.

[7] M. GRomov, Asymptotic invariants of infinite groups. In "Geometric Group Theory”, ed. G. Niblo and M. Roller, Cambridge: Cambridge University Press, (1993).

[8] P. PAnsu, Cohomologie $L_{p}$ et pincement. Comment. Math. Helv., 83 (2008), pp. 327-357.

Manoscritto pervenuto in redazione il 14 marzo 2010. 